\title{
Vacuum-Assisted Incisional Closure Therapy After Groin Reconstruction With Muscle Flap
}

Maryclare E. Taylor ${ }^{1}$, Ledibabari M. Ngaage ${ }^{1}$, Philip Wasicek ${ }^{2}$, Michael Ha ${ }^{1}$, Khanjan Nagarsheth ${ }^{3}$, Shahab A. Toursavadkohi ${ }^{3}$, John Karwowski ${ }^{3}$, Yvonne M. Rasko ${ }^{1}$

1. Plastic and Reconstructive Surgery, University of Maryland School of Medicine, Baltimore, USA 2. General Surgery, University of Maryland School of Medicine, Baltimore, USA 3. Vascular Surgery, University of Maryland School of Medicine, Baltimore, USA

Corresponding author: Yvonne M. Rasko, yrasko@som.umaryland.edu

\section{Abstract \\ Background}

Groin reconstruction with muscle flap coverage is associated with high wound complication rates. Incisional vacuum-assisted closure (iVAC) therapy may lower wound complications. We evaluated the impact of iVAC on postoperative outcomes in patients following groin reconstruction with muscle flap coverage.

\section{Methods}

We conducted a retrospective review of patients who underwent groin reconstruction with muscle flap coverage in 2012-2018. Patients were divided into those who received iVAC therapy and those who received standard sterile dressings (SSD).

\section{Results}

Of the 57 patients included, most received iVAC therapy $(71 \%, n=41)$ and the rest received SSD $(28 \%, n=$ 16). The iVAC group had higher rates of diabetes, hypertension, coronary artery disease, and peripheral artery disease $(p<0.05)$. However, iVAC patients had comparable length of hospital stay (12 vs 8.5 days $p$ $=0.0735)$, reoperations ( $34 \%$ vs $31 \%, p=0.8415)$, and readmissions ( $32 \%$ vs $37 \%, p=0.6801)$ with SSD patients. iVAC placement was less likely in prophylactic flaps (odds ratio $0.08, p=0.0049$ ).

\section{Conclusion}

Patients with a prophylactic flap were less likely to receive vacuum therapy, which may highlight a selection bias where surgeons pre-emptively use iVAC therapy in surgical candidates identified as high risk. The preemptive use of iVAC may minimize adverse postoperative outcomes in high-risk patients.

Review began 02/01/2021 Review ended 04/14/2021 Published 05/11/2021

\section{() Copyright 2021}

Taylor et al. This is an open access article distributed under the terms of the Creative Commons Attribution License CC-BY 4.0., which permits unrestricted use, distribution, and reproduction in any medium, provided the original author and source are credited.
Categories: Plastic Surgery, General Surgery

Keywords: wound closure, wound repair, groin, surgical flaps, negative pressure wound therapy, reconstructive surgical procedures

\section{Introduction}

Wound complications after major surgery on the groin are a serious problem associated with significant morbidity and cost. Complication rates as high as $44 \%$ have been reported in infra-inguinal vascular procedures [1-3]. Surgery on the groin puts a patient at particular risk of infection, wound dehiscence, lymphatic leaks, and hematomas [2,4]. Vascular patients with the presence of comorbidities such as obesity, cigarette use, diabetes, and prior groin surgery are at increased risk of complications [1]. In addition to causing pain and distress to the patient, complications are a significant source of healthcare costs. Surgical site infections (SSIs) incur on average 3.8 additional days in the hospital [5]. Furthermore, the cost of hospitalization in a patient with an SSI was estimated to be $\$ 8,909$ higher than the cost for a patient without an SSI [5-6].

Several methods have been proposed to reduce the complication rates in groin surgery. The use of muscle flaps to treat complications following vascular surgery on the groin was first described in 1989 by Mahoney et al. and has become a widely accepted method of salvage therapy [7-13]. More recently, early use of prophylactic muscle flaps in high-risk patients has been extensively studied and demonstrated to have favorable outcomes $[1,13]$. However, even with prophylactic utilization of muscle flap coverage in high-risk patients, complication rates of $16 \%$ have been reported [1].

Another approach to complication rate reduction in groin surgery is with the use of vacuum-assisted incisional closure therapy (iVAC). The use of iVAC therapy is thought to provide benefits and decrease complication rates by acting as a barrier to the incision from external infectious sources, helping promote 


\section{Cureus}

skin edge apposition, remove fluid and infectious materials from the incision, and increase microcirculation [2,14-18]. A recent meta-analysis of iVAC therapy in patients undergoing groin vascular surgery has shown a statistically significant improvement in outcomes over traditional dressings [6]. Yet, there is no literature examining the use of iVAC therapy on patients with prophylactic or salvage muscle flap groin reconstruction.

The purpose of this study was to delineate the utility of the regular use of iVACs in groin reconstruction via retrospective analysis of patients who have undergone groin reconstruction with muscle flap coverage.

\section{Materials And Methods}

This project was approved by the University of Maryland Institutional Review Board. We performed a retrospective review of all patients who had received groin reconstruction from December 2012 to December 2018. Eligible patients were identified with current procedural terminology (CPT) codes for muscle flap coverage and groin reconstruction (CPT15738 and CPT49568, respectively). An example case is presented in Figure 1. Patients who did not have groin reconstruction but only muscle flap advancement or bilateral abdominal muscle release for ventral hernias were excluded $(n=16)$.

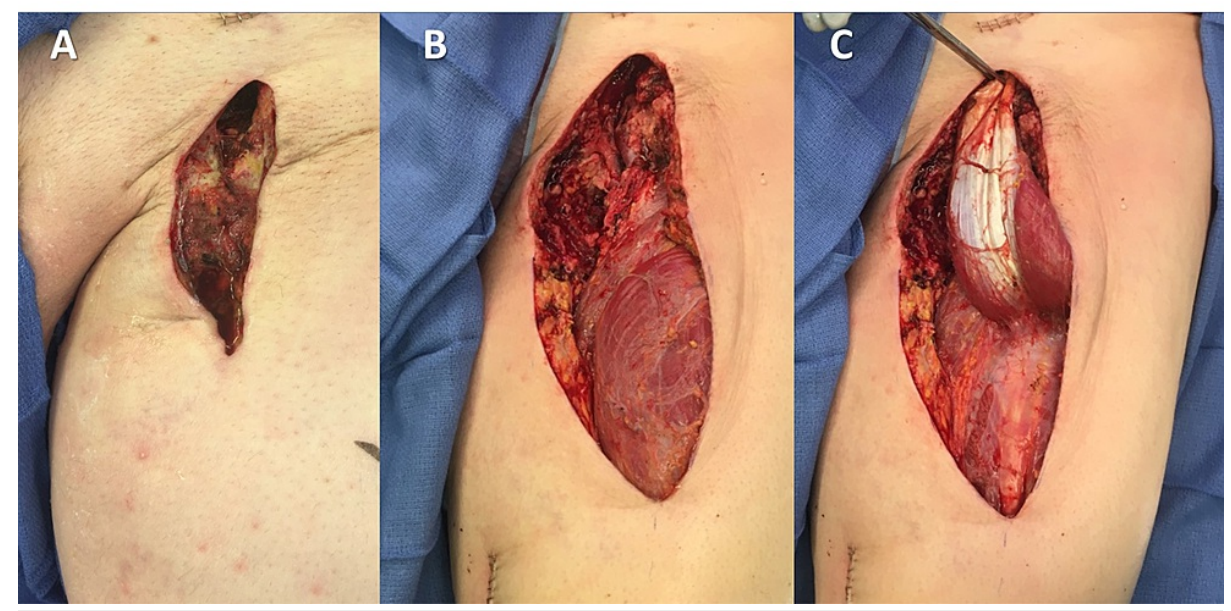

FIGURE 1: Example groin reconstruction via rectus femoris flap

(A) Initial vascular necrotic wound. (B) Exposed bypass graft. (C) Elevation of rectus femoris flap.

Patients were divided into two groups: vacuum-assisted incisional closure (iVAC) (Figure 2) and standard sterile dressing (SSD), which consisted of a dry gauze dressing. We collected details on patient demographics, operative detail, and postoperative course. The primary outcome was postoperative surgical site complications. Surgical site complications included surgical site infections, wound dehiscence, skin necrosis, non-healing incisional wound, seroma, and hematoma. Complications were graded according to the Clavien-Dindo classification [19]. Operative intervention was defined as any surgical site complication that required the opening of the wound, wound debridement, or percutaneous drainage. Secondary outcomes were the length of hospital stay and unplanned readmissions within 90 days. 


\section{Cureus}
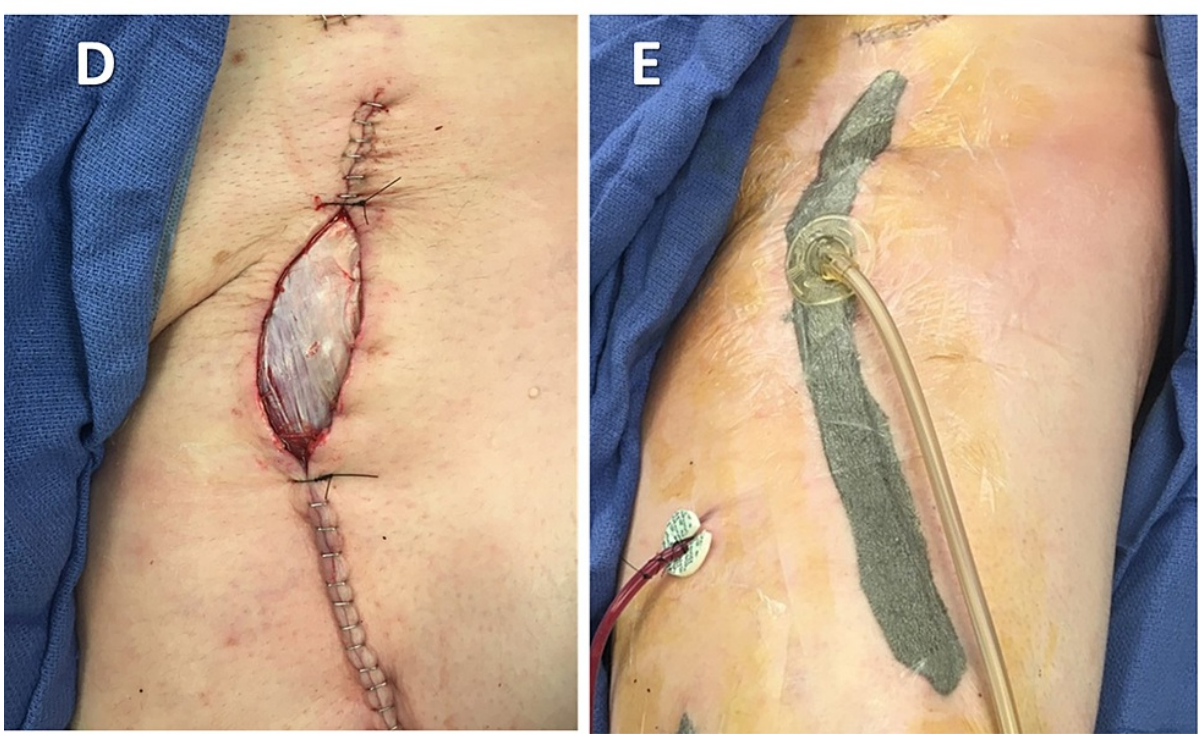

\section{FIGURE 2: Closure of a rectus femoris flap}

(D) Partial closure via staples. (E) Overlaying vacuum-assisted incisional closure therapy (iVAC) dressing.

Composite data were stored in Microsoft Excel (Microsoft Corporation, Redmond, Washington) and analyzed using the Statistical Package for the Social Sciences (SPSS) software version 26 (IBM Corp, 2019. Armonk, $\mathrm{NY)}$. Non-parametric variables were summarized and analyzed using median values and interquartile ranges. Categorical data were summarized using percentages. Differences in continuous data between the groups were tested with the non-parametric Mann-Whitney-U test. The chi-squared test and Fisher exact test were used to test for differences between categorical data, as appropriate. The odds ratio (OR) is reported with $95 \%$ confidence intervals $(95 \% \mathrm{CI})$. Variables found to be significantly different between the two groups in univariate analysis at $\mathrm{p}<0.25$ and clinically relevant variables as determined by clinical judgment were included in the multinomial logistic regression. Variables were checked for multicollinearity (variance inflation factor, VIF) and were excluded if multicollinearity was found (VIF >1.5). Statistical significance was defined as a two-tailed value of $\mathrm{p} \leqslant 0.05$.

\section{Results}

Fifty-seven patients were included in our study. Nearly three quarters received an iVAC (71\%, $\mathrm{n}=41)$ and the rest received an $\operatorname{SSD}(28 \%, \mathrm{n}=16)$. The patient and surgical characteristics of the two study groups are summarized in Table 1. The two study groups were similar in terms of age, gender, body mass index (BMI), and race. However, significant differences in patient comorbidities were observed between the two groups. Patients in the iVAC group had higher rates of diabetes ( $46 \%$ vs $13 \%$, $\mathrm{p}=0.0174$ ), hypertension ( $61 \%$ vs $31 \%$, $\mathrm{p}=0.0434)$, coronary artery disease ( $39 \%$ vs $6 \%, \mathrm{p}=0.0221)$, and peripheral artery disease $(46 \%$ vs $0 \%$, $\mathrm{p}=0.0011$ ) than patients in the SSD group.

\begin{tabular}{|c|c|c|c|c|}
\hline \multicolumn{5}{|l|}{ Patient Demographics } \\
\hline Variable & Total $(n=57)$ & $\operatorname{iVAC}(n=41)$ & $\operatorname{SSD}(n=16)$ & $\mathrm{p}$-value \\
\hline Mean Age / years (SD) & $53.8(17.7)$ & $56.2(18.6)$ & $47.8(13.8)$ & 0.0674 \\
\hline Gender & & & & 0.3801 \\
\hline Male & 34 (60\%) & 26 (63\%) & $8(50 \%)$ & \\
\hline Female & $23(40 \%)$ & 15 (37\%) & $8(50 \%)$ & \\
\hline Mean BMI (SD) & $27.4(7.2)$ & $27.7(7.8)$ & $26.6(5.4)$ & 0.5976 \\
\hline Race & & & & 0.5408 \\
\hline Caucasian & $37(64 \%)$ & $28(69 \%)$ & $9(56 \%)$ & \\
\hline African American & 19 (33\%) & $12(29 \%)$ & $7(44 \%)$ & \\
\hline Other & $1(1 \%)$ & $1(2 \%)$ & $0(0 \%)$ & \\
\hline
\end{tabular}




\section{Cureus}

Medical history

Diabetes

CAD

PAD

HTN

Cigarette use

Previous groin surgery

Timing of flap

Salvage

Prophylactic

Operative indication

Infection

Hemorrhage

Oncologic recon

Orthopedic recon

Trauma recon

Vascular recon

IBD recon

Flap type

Gracilis

Rectus femoris

Sartorius

Tensor fascia lata

Fasciocutaneous

Gastrocnemous

Soleus

Vastus lateralis

Vastus medialis

Other

Median follow-up time / days (Q1, Q3)

Mean follow-up time / days (SD)
$21(37 \%)$

$19(46 \%)$

$2(13 \%)$

0.0174

17 (30\%)

16 (39\%)

$1(6 \%)$

0.0221

19 (46\%)

$0(0 \%)$

0.0011

30 (53\%)

25 (61\%)

$5(31 \%)$

0.0434

45 (79\%)

35 (85\%)

$10(63 \%)$

0.0762

44 (77\%)

35 (85\%)

$9(56 \%)$

0.0327

$35(60 \%)$

32 (78\%)

$3(19 \%)$

$<0.0001$

23 (40\%)

$9(21 \%)$

$13(81 \%)$

27 (47\%)

$26(63 \%)$

$1(6 \%)$

$1(2 \%)$

11 (19\%)

$1(2 \%)$

$0(0 \%)$

2 (5\%)

$9(56 \%)$

$5(9 \%)$

$5(12 \%)$

$0(0 \%)$

$1(2 \%)$

$1(6 \%)$

$6(15 \%)$

$1(6 \%)$

$0(0 \%)$

$4(25 \%)$

4 (7\%)

15 (26\%)

$2(4 \%)$

$13(81 \%)$

$14(24 \%)$

$13(31 \%)$

$1(6 \%)$

17 (41\%)

$0(0 \%)$

$1(2 \%)$

$0(0 \%)$

$1(2 \%)$

$1(2 \%)$

$0(0 \%)$

$1(6 \%)$

$1(2 \%)$

$1(2 \%)$

$1(6 \%)$

$1(2 \%)$

$1(2 \%)$

$0(0 \%)$

$7(12 \%)$

$6(15 \%)$

$1(6 \%)$

$1(2 \%)$

$1(2 \%)$

$0(0 \%)$

4 (7\%)

$3(7 \%)$

$1(6 \%)$

$547(67,836.5)$

$399(44,819.5)$

$679(91,919.5)$

0.0093

$513.7(453.1)$

$402.5(387.5)$

0.0023

\section{TABLE 1: Patient demographics}

CAD, coronary artery disease; PAD, peripheral artery disease; HTN, hypertension; IBD, inflammatory bowel disease; BMI, body mass index; SSD, standard sterile dressing; iVAC, vacuum-assisted incisional closure device; SD, standard deviation; Q1; Q3, first quartile, third quartile; recon, reconstruction

A greater proportion of patients in the iVAC group had a history of prior groin surgery as compared to the SSD group ( $85 \%$ vs $56 \%, \mathrm{p}=0.0327$ ). Additionally, $78 \%$ of patients who underwent iVAC placement had salvage, rather than prophylactic, muscle flaps as compared to $19 \%$ of patients in the SSD group with salvage muscle flaps $(\mathrm{p}<0.0001)$. There were increased odds of iVAC use with salvage flaps (OR=15.41, 95\% CI: 3.59- 


\section{Cureus}

66.15, $\mathrm{p}=0.0002)$. Groin reconstruction for infective indication was more common in patients who underwent iVAC placement than the SSD group (63\% vs 6\%, p<0.0001), whereas oncologic reconstruction was significantly more prevalent in the SSD group ( $56 \%$ vs $5 \%$, p $<0.0001)$. The most common muscle flaps utilized in the iVAC group were the sartorius $(29 \%, n=17)$, gracilis $(28 \%, n=16)$, and rectus femoris $(31 \%$, $\mathrm{n}=13)$, whereas the SSD group almost exclusively received a gracilis muscle flap $(81 \%, \mathrm{n}=13)$.

The comparison of postoperative outcomes is shown in Table 2. The mean operative time for patients with iVAC placement was significantly less than for patients receiving SSD (199.6 minutes vs 364 minutes, $\mathrm{p}<0.0001$ ). There was no significant difference in the number of patients who received postoperative blood transfusions between the iVAC and SSD groups (20\% vs 19\%, p=1.0000). Patients with iVAC placement had comparable median hospital stays to those in the SSD group (12 vs $8.5, \mathrm{p}=0.0735$ ). There was also no significant difference between the iVAC and SSD rates of surgical site complications (39\% vs $31 \%$, $\mathrm{p}=0.5838$ ). Similarly, the iVAC and SSD groups had similar rates of operative interventions to treat complications ( $34 \%$ vs $31 \%$, p=0.8415) and readmissions within 90 days (32\% vs 37\%, p=0.6801).

\begin{tabular}{|c|c|c|c|c|}
\hline \multicolumn{5}{|l|}{ Perioperative characteristics } \\
\hline Variable & Total $(n=57)$ & $\operatorname{iVAC}(n=41)$ & $\operatorname{SSD}(n=16)$ & $\mathrm{p}$-value \\
\hline Mean operative time / minutes (SD) & 246.6 (147.5) & $199.6(115.7)$ & 364 (154.9) & $<0.0001$ \\
\hline Patients who received blood transfusion & $11(16 \%)$ & $8(20 \%)$ & $3(19 \%)$ & 1.0000 \\
\hline Median length of hospital stay / days (Q1, Q3) & $11(7,20)$ & $12(8,21)$ & $8.5(5.75,12)$ & 0.0735 \\
\hline Surgical site complications & $21(37 \%)$ & $16(39 \%)$ & $5(31 \%)$ & 0.5838 \\
\hline Infection & $7(12 \%)$ & $3(7 \%)$ & $4(25 \%)$ & \\
\hline Wound dehiscence & $4(7 \%)$ & $2(5 \%)$ & $2(13 \%)$ & \\
\hline Skin necrosis & $3(5 \%)$ & $2(5 \%)$ & $1(6 \%)$ & \\
\hline Chronic wound & $3(5 \%)$ & $2(5 \%)$ & $1(6 \%)$ & \\
\hline Seroma & $2(4 \%)$ & $2(5 \%)$ & $0(0 \%)$ & \\
\hline Hematoma & $3(5 \%)$ & $3(7 \%)$ & $0(0 \%)$ & \\
\hline Other & $2(\%)$ & $2(5 \%)$ & $0(0 \%)$ & \\
\hline 90-day operative intervention & $19(33 \%)$ & $14(34 \%)$ & $5(31 \%)$ & 0.8415 \\
\hline Readmissions & $19(33 \%)$ & $13(32 \%)$ & $6(37 \%)$ & 0.6801 \\
\hline
\end{tabular}

\section{TABLE 2: Perioperative characteristics}

SSD, standard sterile dressing; iVAC, vacuum-assisted incisional closure device; SD, standard deviation; Q1; Q3, first quartile, third quartile

Based on univariate analysis and clinical judgment, we identified variables of interest for multinomial logistic regression. Infection as an operative indication was found to be collinear with salvage flaps $(\mathrm{VIF}=$ 2.27) so was excluded from the analysis. We assessed risk factors for surgical site complications, reoperations, and readmissions (Table 3). The use of iVAC therapy did not significantly change rates of complications (OR=0.86, 95\% CI: 0.16-4.62, $\mathrm{p}=0.8630$ ), 90-day reoperations (OR=0.52, 95\% CI: 0.08-3.52, $\mathrm{p}=0.5044)$, or 90 -day readmissions $(\mathrm{OR}=0.85$, 95\% CI: $0.15-5.01, \mathrm{p}=0.8581)$. However, diabetes was shown to be a risk factor for surgical site complications (OR=5.52, 95\% CI: 1.13-27.17, $\mathrm{p}=0.0356$ ) and 90-day reoperations $(\mathrm{OR}=18.1,95 \% \mathrm{CI}: 2.38-137.25, \mathrm{p}=0.0051)$. Patients with a previous groin surgery had lower odds of experiencing a complication that required operative intervention (OR=0.10, 95\% CI: 0.01-0.94, $\mathrm{p}=0.0437)$. 


\section{Cureus}

Multivariate analysis for risk factors for surgical site complications

\begin{tabular}{|c|c|c|c|c|c|c|}
\hline \multirow[t]{2}{*}{ Variable } & \multicolumn{2}{|c|}{ Surgical site complication } & \multicolumn{2}{|l|}{ 90-day reoperations } & \multicolumn{2}{|c|}{ 90-day readmissions } \\
\hline & OR $(95 \% \mathrm{Cl})$ & $\mathrm{p}$-value & OR $(95 \% \mathrm{Cl})$ & $\mathrm{p}$-value & OR $(95 \% \mathrm{Cl})$ & $\mathrm{p}$-value \\
\hline iVAC & $0.86(0.16-4.62)$ & 0.8630 & $0.52(0.08-3.52)$ & 0.5044 & $0.85(0.15-5.01)$ & 0.8581 \\
\hline \multicolumn{7}{|l|}{ Medical history } \\
\hline Diabetes & $5.52(1.13-27.17)$ & 0.0356 & $18.1(2.38-137.25)$ & 0.0051 & $5.11(0.86-30.46)$ & 0.0735 \\
\hline CAD & $0.66(0.11-4.13)$ & 0.6552 & $0.34(0.04-2.68)$ & 0.3033 & $2.91(0.39-21.62)$ & 0.2977 \\
\hline PAD & $0.63(0.08-4.94)$ & 0.6652 & $0.85(0.08-9.24)$ & 0.8928 & $0.45(0.05-3.90)$ & 0.4685 \\
\hline HTN & $1.77(0.33-9.52)$ & 0.5081 & $0.91(0.12-7.19)$ & 0.9302 & $0.79(0.14-4.52)$ & 0.7904 \\
\hline Smoking & $6.42(0.77-53.82)$ & 0.0863 & 7.67 (0.75 - 78.53) & 0.0860 & $5.72(0.68-47.79)$ & 0.1074 \\
\hline Previous groin surgery & $0.42(0.07-2.64)$ & 0.3560 & $0.10(0.01-0.94)$ & 0.0437 & $0.95(0.16-5.68)$ & 0.9590 \\
\hline Salvage flap & $1.00(0.18-5.71)$ & 1.0000 & $2.43(0.29-20.03)$ & 0.4096 & $0.23(0.04-1.50)$ & 0.1241 \\
\hline
\end{tabular}

TABLE 3: Multivariate analysis for risk factors for surgical site complications

CAD, coronary artery disease; Cl, confidence interval; HTN, hypertension; iVAC, incisional vacuum-assisted closure therapy; OR, odds ratio; PAD, peripheral artery disease

\section{Discussion}

The aim of this study was to evaluate the utility of iVAC use in patients with muscle flap reconstruction of the groin. Our results show that iVAC therapy in groin reconstruction with muscle flap has comparable outcomes to standard sterile dressings despite use in a higher risk patient population. Furthermore, diabetes mellitus is the most important predictor for postoperative complications and reoperations within this patient population.

In our patient population, patients who received iVAC therapy were more likely to have risk factors such as diabetes, hypertension, coronary artery disease, and peripheral artery disease. Our results demonstrate that diabetes was an independent risk factor for surgical site complications and 90-day reoperations, which is consistent with previous literature [1,20-21]. Additionally, iVAC use was more common in patients receiving free flaps for infective indications. These patients are often critically ill, require long-term antibiotics, and have been found to have mortality rates ranging from $6 \%$ to $75 \%$ [22]. The decision to utilize iVAC therapy was surgeon-dependent. iVAC therapy is thought to improve wound healing by creating a protective barrier, removing fluid and infectious materials from the incision, providing a better approximation of wound edges with decreased lateral tissue tension, and a potential increase in micro-circulation and oxygen saturation [2,14-18]. Furthermore, previous studies have found iVAC use to be associated with lower rates of surgical site infections than traditional dressings in high-risk patients [2,20,23]. Knowledge of this data may have led to a surgeon selection bias in which iVAC therapy was preferentially used in patients considered higher risk.

Additionally, patients were more likely to receive iVAC therapy in salvage groin reconstruction surgery, rather than prophylactically. The use of muscle flap as salvage therapy in wound complications of the groin has a well-studied benefit due to increased vascularization of the complication site, elimination of wound dead space, and increased lymphatic drainage around the site of transposition [1,7-10,12-13]. More recently, studies have proposed that the use of prophylactic muscle flaps may decrease complication rates in groin reconstruction [1]. The increased use of iVAC therapy in patients undergoing salvage muscle flap procedures may be secondary to surgeon bias. Our data reflects this bias, as the patients who underwent iVAC therapy were more likely to have groin flap reconstruction due to infectious indications.

Multivariate analysis demonstrated that iVAC did not influence surgical site complications, 90-day reoperations, or 90 -day readmissions. This calls into question whether iVAC, which can cost up to $\$ 495$, conveys a significant enough benefit to outweigh the inexpensive standard sterile dressing [2]. Further studies with larger patient populations and randomization of therapy as well as cost-benefit analyses are necessary to elicit the impact of iVAC therapy on groin complications and the economic utility of this therapy. 
There are several limitations to our study. A major limitation is the introduction of selection bias, as patients were not randomized to iVAC vs SSD and the use of iVAC-assisted therapy was based on the surgeon's discretion. Patients who were at higher preoperative risk were more likely to receive iVAC therapy. This is evidenced by the higher rates of diabetes, coronary artery disease, peripheral artery disease, and hypertension in the iVAC group. These factors may account for comparable outcomes between the iVAC and SSD groups. Patients with a prophylactic flap were less likely to receive iVAC therapy, which may further highlight selection bias in which surgeons pre-emptively use iVAC therapy in patients with pre-existing wound complications. This study also included patients treated by several different surgeons in varying surgical specialties, including general surgery, plastic surgery, and vascular surgery. Thus, inter-operator variability may be a confounding factor that was not accounted for in our analysis.

\section{Conclusions}

Patients who received iVAC therapy had comparable complication, reoperation, and readmission rates to those who received standard dressings despite the possible higher preoperative risk for complications amongst the iVAC therapy group. The use of vacuum-assisted incisional closure therapy may be linked to a selection bias by the surgeon and used preferentially in patients perceived to be at high risk for complications after muscle flap groin reconstruction. The pre-emptive use of iVAC may minimize adverse postoperative outcomes in high-risk patients.

\section{Additional Information}

\section{Disclosures}

Human subjects: Consent was obtained or waived by all participants in this study. University of Maryland Institutional Review Board issued approval HP-00083316. Animal subjects: All authors have confirmed that this study did not involve animal subjects or tissue. Conflicts of interest: In compliance with the ICMJE uniform disclosure form, all authors declare the following: Payment/services info: All authors have declared that no financial support was received from any organization for the submitted work. Financial relationships: All authors have declared that they have no financial relationships at present or within the previous three years with any organizations that might have an interest in the submitted work. Other relationships: All authors have declared that there are no other relationships or activities that could appear to have influenced the submitted work.

\section{References}

1. Fischer JP, Nelson JA, Rohrbach JI, et al.: Prophylactic muscle flaps in vascular surgery. The Penn Groin Assessment Scale. Plast Reconstr Surg. 2012, 129:940e-9. 10.1097/PRS.0b013e31824ecb17

2. Matatov T, Reddy KN, Doucet LD, Zhao CX, Zhang WW: Experience with a new negative pressure incision management system in prevention of groin wound infection in vascular surgery patients. J Vasc Surg. 2013, 57:791-5. 10.1016/j.jvs.2012.09.037

3. Kent KC, Bartek S, Kuntz KM, Anninos E, Skillman JJ: Prospective study of wound complications in continuous infrainguinal incisions after lower limb arterial reconstruction: incidence, risk factors, and cost. Surgery. 1996, 119:378-383. 10.1016/s0039-6060(96)80135-8

4. Centers for Disease Control and Prevention. 2019 National and State Healthcare-Associated Infections Progress Report. Atlanta, GA. http://www.cdc.gov/hai/surveillance/progress-report/index.html.

5. Hatoum HT, Akhras KS, Lin SJ: The attributable clinical and economic burden of skin and skin structure infections in hospitalized patients: a matched cohort study. Diagn Microbiol Infect Dis. 2009, 64:305-10. 10.1016/j.diagmicrobio.2009.02.005

6. Singh DP, Gabriel A, Parvizi J, Gardner MJ, D'Agostino R Jr: Meta-analysis of comparative trials evaluating a single-use closed-incision negative-pressure therapy system. Plast Reconstr Surg. 2019, 143:41S-46S. 10.1097/PRS.0000000000005312

7. Mahoney J: Salvage of the infected groin vascular graft with muscle flaps . Ann Plast Surg. 1989, 22:252-6. 10.1097/00000637-198903000-00012

8. Morasch MD, Sam AD 2nd, Kibbe MR, Hijjawi J, Dumanian GA: Early results with use of gracilis muscle flap coverage of infected groin wounds after vascular surgery. J Vasc Surg. 2004, 39:1277-83. 10.1016/j.jvs.2004.02.011

9. Shermak MA, Yee K, Wong L, Jones CE, Wong J: Surgical management of groin lymphatic complications after arterial bypass surgery. Plast Reconstr Surg. 2005, 115:1954-62. 10.1097/01.prs.0000165069.15384.e5

10. May BL, Zelenski NA, Daluvoy SV, Blanton MW, Shortell CK, Erdmann D: Salvage of exposed groin vascular grafts with early intervention using local muscle flaps. Plast Reconstr Surg Glob Open. 2015, 3:e514. 10.1097/GOX.0000000000000480

11. Dua A, Rothenberg KA, Lavingia K, Ho VT, Rao C, Desai SS: Outcomes of gracilis muscle flaps in the management of groin complications after arterial bypass with prosthetic graft. Ann Vasc Surg. 2018, 51:1138. 10.1016/j.avsg.2018.02.009

12. Colwell AS, Donaldson MC, Belkin M, Orgill DP: Management of early groin vascular bypass graft infections with sartorius and rectus femoris flaps. Ann Plast Surg. 2004, 52:49-53. 10.1097/01.sap.0000099960.68038.53

13. Brewer MB, Ochoa CJ, Woo K, et al.: Sartorius muscle flaps for vascular groin wound complications . Am Surg. 2015, 81:1163-9.

14. Diaconu SC, McNichols CHL, Ngaage LM, et al.: Closed-incision negative-pressure therapy decreases complications in ventral hernia repair with concurrent panniculectomy. Hernia. 2020, 24:49-55. 


\section{Cureus}

10.1007/s10029-018-1865-2

15. Atkins BZ, Tetterton JK, Petersen RP, Hurley K, Wolfe WG: Laser Doppler flowmetry assessment of peristernal perfusion after cardiac surgery: beneficial effect of negative pressure therapy. Int Wound J. 2011, 8:56-62. 10.1111/j.1742-481X.2010.00743.x

16. Dohmen PM, Misfeld M, Borger MA, Mohr FW: Closed incision management with negative pressure wound therapy. Expert Rev Med Devices. 2014, 11:395-402. 10.1586/17434440.2014.911081

17. Kilpadi DV, Lessing C, Derrick K: Healed porcine incisions previously treated with a surgical incision management system: mechanical, histomorphometric, and gene expression properties. Aesthetic Plast Surg. 2014, 38:767-78. 10.1007/s00266-014-0339-x

18. Wilkes RP, Kilpad DV, Zhao Y, Kazala R, McNulty A: Closed incision management with negative pressure wound therapy (CIM): biomechanics. Surg Innov. 2012, 19:67-75. 10.1177/1553350611414920

19. Dindo D, Demartines N, Clavien PA: Classification of surgical complications: a new proposal with evaluation in a cohort of 6336 patients and results of a survey. Ann Surg. 2004, 240:205-13. 10.1097/01.sla.0000133083.54934.ae

20. Zaidi A, El-Masry S: Closed-incision negative-pressure therapy in high-risk general surgery patients following laparotomy: a retrospective study. Colorectal Dis. 2017, 19:283-7. 10.1111/codi.13458

21. Inui T, Bandyk DF: Vascular surgical site infection: risk factors and preventive measures . Semin Vasc Surg. 2015, 28:201-7. 10.1053/j.semvascsurg.2016.02.002

22. Wübbeke LF, Elshof JW, Conings JZM, Scheltinga MR, Daemen JHC, Mees BME: A systematic review on the use of muscle flaps for deep groin infection following vascular surgery. J Vasc Surg. 2020, 71:693-700.e1. 10.1016/j.jvs.2019.07.073

23. Lee K, Murphy PB, Ingves MV, et al.: Randomized clinical trial of negative pressure wound therapy for highrisk groin wounds in lower extremity revascularization. J Vasc Surg. 2017, 66:1814-9.

10.1016/j.jvs.2017.06.084 